\title{
Medium-Distance GPS Ambiguity Resolution with Controlled Failure Rate
}

\author{
D. Odijk, S. Verhagen, and P.J.G. Teunissen
}

\begin{abstract}
The goal of Network RTK is to provide users with precise ionospheric corrections in order to conduct fast GPS ambiguity resolution and to get $\mathrm{cm}$-level positioning results over medium-distance baselines. In this paper it is shown that a Network RTK user should apply the ratio test with fixed failure rate, having a threshold value that depends on the model at hand, as to test whether the estimated integer solution can be accepted with sufficient more likelihood than the second-best integer solution. Application of the traditional ratio test (with a fixed threshold value) may namely result in too many wrong fixes and consequently severe positioning errors. However, in the paper it is also demonstrated that the ratio test with fixed failure rate should be applied with care, since its correct performance depends on the correctness of the underlying model.
\end{abstract}

\subsection{Introduction}

Carrier phase ambiguity resolution is definitely the key to fast and rapid GNSS positioning. It is well known that the distance between rover and reference receiver is a limiting factor for these RTK applications, since for medium baselines of tens of

D. Odijk

Department of Spatial Sciences, Curtin University of Technology, Perth, Australia

S. Verhagen $(\bowtie)$

Delft Institute of Earth Observation and Space Systems (DEOS), Delft University of Technology, Delft, The Netherlands e-mail: a.a.verhagen@tudelft.nl

P.J.G. Teunissen

Department of Spatial Sciences, Curtin University of Technology, Perth, Australia

Delft Institute of Earth Observation and Space Systems (DEOS), Delft University of Technology, Delft, The Netherlands kilometers (and longer) differential ionospheric and tropospheric errors may seriously hamper successful ambiguity resolution. To solve for the ionosphere, in many parts of the world permanent GNSS networks have been set up: using the data of these reference stations and based on the smooth spatial behavior of the ionosphere the relative ionospheric delays can be predicted (interpolated) and disseminated to users in the form of ionospheric corrections. The tropospheric errors are usually dealt with by the user by estimating a zenith tropospheric delay parameter. The sketched technique is known as Network RTK, see e.g. (Vollath et al. 2000), or Wide Area RTK (Hernández-Pajares et al. 2004). The success of this technique largely depends on the quality of the ionospheric corrections: when they would perfectly match the true (unknown) ionospheric delays, the probability of successful ambiguity resolution will be close to 1 , however if residual ionospheric delays remain present, a lower success rate may be expected. As part of the ambiguity 
resolution procedure in GPS RTK practice usually a ratio test is carried out, in order to test whether the integer solution may be accepted or not.

In this paper the performance of the ratio test with fixed failure rate (the so-called FFRatio test, see Teunissen and Verhagen (2009)) is investigated. In contrast to traditional ratio tests, the FFRatio test has the advantage that the user has a priori control of the probability of wrong ambiguity fixing. The FFRatio test will be applied in combination with the LAMBDA method for baselines up to $40 \mathrm{~km}$, for which ionospheric corrections are available from a network of permanent stations. The paper is set up as follows. Section 93.2 reviews the ionosphere-weighted model, which forms the starting point of medium-distance GPS ambiguity resolution. In Sect. 93.3 the applied ambiguity resolution procedure is briefly described, while test results are presented in Sect. 93.4. Finally, in Sect. 93.5 the conclusions of the paper are presented.

\subsection{The lonosphere-Weighted GPS Model}

For medium-distance GPS baselines the differential ionospheric delays cannot be neglected, but need to be incorporated into the observation equations. As a more general formulation, we consider the ionospheric delays as stochastic variables, which can be constrained or weighted in the processing. The ionosphere-weighted model of GPS observation equations then reads (Odijk 1999):

$E\left(\left[\begin{array}{c}w \\ i\end{array}\right]\right)=\left[\begin{array}{ccc}G & C & M \\ 0 & I & 0\end{array}\right]\left[\begin{array}{c}g \\ i \\ a\end{array}\right] ; D\left(\left[\begin{array}{c}w \\ i\end{array}\right]\right)=\left[\begin{array}{cc}Q_{w} & 0 \\ 0 & Q_{i}\end{array}\right]$

with $E($.$) the expectation and D($.) the dispersion operator. Vector $w$ contains the observed-minus-computed double difference (DD) code and carrier phase observations, while vector $i$ represents the DD ionospheric delays. At the parameter side, vector $g$ contains the unknown baseline components and -if necessary- tropospheric (zenith) delays, while the unknown integer ambiguities are captured by vector $a$. Matrices $G, C, M$ and $I$ (with $I$ the identity matrix) capture the functional relations between the observations and parameters. The precision of the DD code and phase observations is described by the variance matrix $Q_{w}$, while $Q_{i}$ denotes the variance matrix of the ionospheric observations or constraints. The inverse of this latter matrix can be considered as ionospheric weight matrix, hence the model is referred to as the ionosphere-weighted model. As extremes of the ionosphere-weighted model, if we set $Q_{i}=0$, we have the ionosphere-fixed model (the traditional shortbaseline model), in which the ionospheric variables are not unknown but deterministic, whereas if $Q_{i}=\infty$, the ionosphere-float model is obtained, in which the ionospheric variables are considered as completely unknown parameters (the long-baseline model).

\subsection{Multi-Carrier Ambiguity Resolution}

The integer ambiguities are estimated using the integer least-squares algorithm implemented in the LAMBDA method (Teunissen 1995). Mathematically, the integer estimation can be described using the following projection $S: \mathbb{R}^{n} \mapsto \mathbb{Z}^{n}$ :

$$
\check{a}=S(\hat{a})
$$

with $\hat{a}$ the float ambiguity vector and $\breve{a}$ the integer vector. Input for the LAMBDA method are the float ambiguity vector plus its variance matrix, denoted as $Q_{\hat{a}}$.

To accept the integer least-squares ambiguity solution as obtained by the LAMBDA method, the Fixed Failure-rate (FF) Ratio test (Teunissen and Verhagen 2009) is executed, in order to test whether the integer solution is sufficiently more likely than the secondbest solution. The integer least-squares solution $\check{a}$ is only accepted if and only if:

$$
\frac{\|\hat{a}-\check{a}\|_{Q_{\hat{a}}}^{2}}{\left\|\hat{a}-\check{a}_{2}\right\|_{Q_{\hat{a}}}^{2}} \leqslant \mu
$$

with the squared norm defined as $\|\cdot\|_{Q}^{2}=(\cdot)^{T} Q^{-1}(\cdot)$ and where $\check{a_{2}}$ is the second-best integer ambiguity solution and $\mu$ the critical value of the ratio test. If the FFRatio test is not passed, the integer solution should not be used, and one has to be satisfied with the float solution. 
The FFRatio test differs from the more traditional ratio test, see e.g. (Leick 2003), by the choice of the threshold value $\mu$. In traditional ratio tests, this value is set to a fixed value (e.g. $1 / 2$ or $1 / 3$ ), irrespective of the model at hand. Using the FFRatio test, the threshold value depends on the GNSS model at hand $(0<\mu \leq 1)$. As shown by Teunissen and Verhagen (2009), using the FFRatio test it is guaranteed that the probability of wrong integer estimation (i.e. the failure rate) is lower than a fixed user defined threshold (e.g. 0.001), provided that the underlying models are correct. A user however does not have control of the failure rate when using the ratio test with fixed threshold value. The model-driven threshold value, as we use for this paper, depends on the actual variance matrix of the float ambiguities $Q_{\hat{a}}$ and a practical approach to determine it is by using look-up tables, generated by simulations, giving the threshold value $\mu$ as function of a certain integer least-squares failure rate for varying $n$ (number of ambiguities), see Teunissen and Verhagen (2009). The integer least-squares failure rate is here defined as 1 minus the integer least-squares success rate, which can be approximated by computing the success rate of integer bootstrapping based on the conditional standard deviations of the LAMBDAdecorrelated ambiguities (Teunissen 2005).

\subsection{Test Results}

As source of test data we have used GPS data of the Southern California Integrated GPS Network (SCIGN). This dense permanent GPS network consists of high-end dual-frequency GPS receivers, of which the data are freely available through the Internet (SCIGN 2008). From this network we selected four GPS receivers (i.e. NOPK-VNPS-CLAR-OAT2), acting as permanent network from which ionospheric corrections are generated, see Fig. 93.1. The master reference station of this network is station NOPK. As can be seen from the figure, the maximum distance between these reference stations is approximately $70 \mathrm{~km}$. and OAT2 at $1,113 \mathrm{~m}$. The four GPS receivers are all Ashtech Z-XII3 geodetic receivers.

The two SCIGN stations FXHS and WLSN (also equipped with Ashtech Z-XII3 receivers) have been selected as two rovers stations as to test the performance of network RTK. The baseline NOPK-FXHS is $11 \mathrm{~km}$, so we refer to this as a short baseline, while the

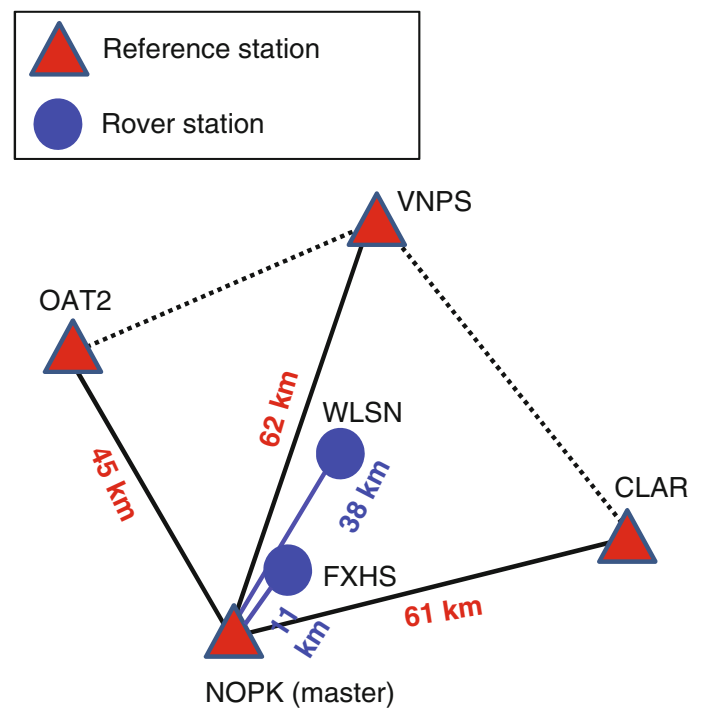

Fig. 93.1 Simulated permanent network and rover stations

$38-\mathrm{km}$ baseline NOPK-WLSN is referred to as a medium baseline.

GPS data of 1 January 2000 were selected as test data set. This date falls in a period of reasonable high solar activity, where the planetary Kp index, a measure for geomagnetic activity affecting the ionospheric activity, has increased levels $(\mathrm{Kp}>4)$ for some part of the day. The maximum value the Kp can take on, is 9 .

\subsubsection{Permanent Network Processing}

The GPS data of the four permanent stations have been processed using in-house software based on a Kalman filter implementation. Because of its recursive character, this Kalman filter is suitable for real-time processing. In the time update of the filter it is assumed that the DD ambiguities are constant from one epoch to the next (provided that no cycle slips occur). The GPS data of the four receivers are processed using a true network solution (taking the mathematical correlations into account) of the ionosphere-weighted model, using the following settings:

- Dual-frequency phase and code data (L1, L2, C1, P2)

- Data sampling: $30 \mathrm{~s}$; thus 2,880 epochs

- Cut-off elevation: $10^{\circ}$; there are 5-10 satellites in view during the day 
- Standard deviation phase: $2 \mathrm{~mm}$, code: $20 \mathrm{~cm}$ (undifferenced; in local zenith)

- Ionospheric observations with zero values and standard deviations of $10 \mathrm{~cm}$ (undifferenced)

- Observations are elevation-dependent weighted using an exponential function

- No parameterization of receiver positions

- Parameterization of one zenith tropospheric delay per network station

- Satellite positions based on precise predicted IGS orbits

- Integer ambiguities are estimated after 30 epochs of initializing the filter and also after 30 epochs for the integer ambiguities of a newly risen satellite

Figure 93.2 shows the DD ionospheric delays, based on the fixed integer ambiguities, for the three 'baselines' in the network (all relative to NOPK). From the figures the daily cycle of the ionosphere is clearly visible: during local night time the DD ionospheric delays are small $(\mathrm{few} \mathrm{cm})$, while during day time these may rise up to $8-10 \mathrm{~mm}$ per $\mathrm{km}$ baseline length. Another feature of the ionosphere that can be seen in the figures is the strong spatial correlation between the DD ionospheric delays of the three baselines. The presence of this spatial correlation is crucial for the performance of network RTK since the ionospheric corrections for the rover receivers are interpolated (based on Kriging) from these spatially correlated network DD ionospheric delays.

\subsubsection{Rover's Baseline Processing}

Ionospheric corrections have been generated based on the approximate locations of the two rover stations, FXHS (at $11 \mathrm{~km}$ from NOPK) and WLSN (at $38 \mathrm{~km}$ from NOPK). The GPS data of these two rover stations are processed as independent baselines both having NOPK as reference. The ionosphere-weighted model serves as basis of the processing of the rover data, having the network ionospheric corrections as ionospheric observations (see (1)). The choice of the ionospheric standard deviation (modeled in $Q_{i}$ ) is crucial here. It should match the quality of the ionospheric corrections: a low standard deviation if the corrections closely resemble the true ionospheric delays, and a higher standard deviation if discrepancies can be expected between interpolated and true delays. The discrepancies between true and interpolated delays are basically a function of the state of the ionosphere, the distances between the network stations and the length of the rover baseline. The baseline length dependence is confirmed for the two rover baselines, see Fig. 93.3, which shows the DD ionospheric delays estimated from the GPS data themselves and the residuals after applying the network ionospheric corrections. It can be seen that after applying the corrections for the $11-\mathrm{km}$ baseline the (absolute) residuals are all below $5 \mathrm{~cm}$, but for the $38-\mathrm{km}$ baseline these can be up to $12 \mathrm{~cm}$. It is emphasized that the ionospheric residuals could be computed here since we know the true ionospheric delays (from postprocessing). For truly real-time applications an approach to assess the quality of the ionospheric corrections is to predict the ionosphere not only for the rover but also for a reference station that is within the coverage of the network but not included in the correction generation. For such a station the differences between predicted and true ionospheric delays can be continuously monitored and information on the quality of the ionospheric corrections can be determined and disseminated to rovers. For this paper the ionospheric
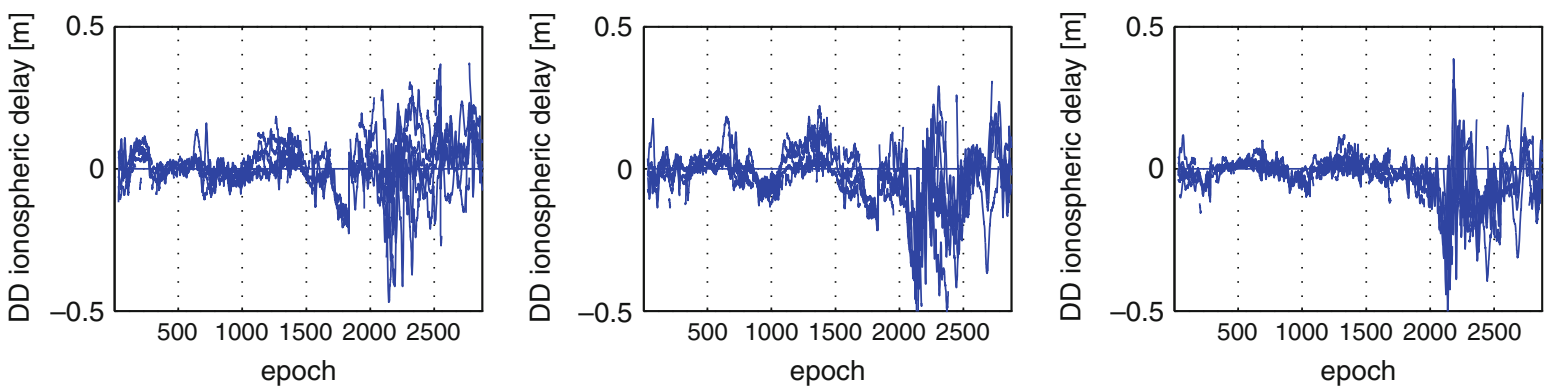

Fig. 93.2 Ambiguity-fixed DD ionospheric delays for all satellites in the permanent network data: (left) 61-km baseline NOPKCLAR, (middle) 62-km baseline NOPK-VNPS, (right) 45-km baseline NOPK-OAT2 
Fig. 93.3 Ambiguity-fixed DD ionospheric delays in short (11-km; top) and medium (38-km; bottom) baselines: without any correction (left) and after subtracting the network corrections (right)
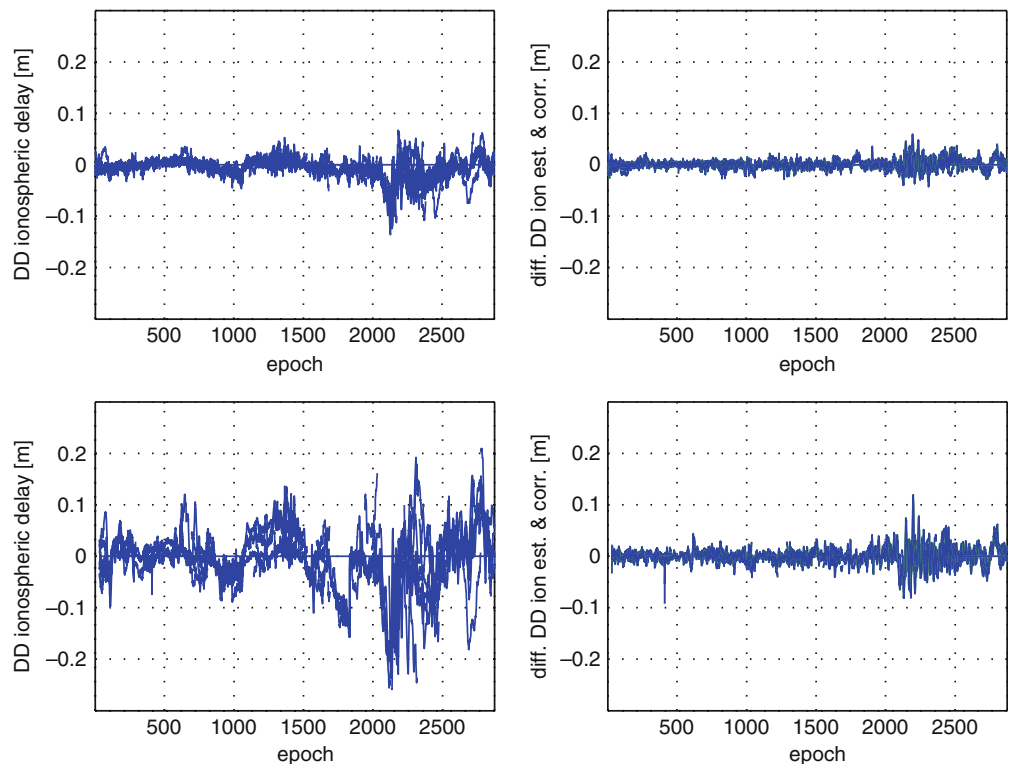

standard deviations empirically derived from the residuals are $5 \mathrm{~mm}$ for the short baseline and $1 \mathrm{~cm}$ for the medium baseline (both values are undifferenced and apply to zenith).

For both baselines the rover processing will be carried out on an epoch-by-epoch basis, as to investigate the performance of the fastest method of ambiguity resolution. Thus the ambiguities are resolved instantaneously (using LAMBDA and the FFRatio test), based on only the data of the current epoch, without taking any information of previous epochs into account. In all computations the failure rate of the FFRatio test has been set to 0.001 (so for $0.1 \%$ of the epochs a wrong integer solution is expected).

\subsubsection{Short-Baseline Performance}

As the traditional GPS processing model for short baselines is based on completely neglecting the differential ionospheric delays, in addition to the ionosphere-weighted processing, the $11-\mathrm{km}$ baseline data are also processed using the ionosphere-fixed model, in absence of using any ionospheric corrections. The same settings are applied to the ionosphere-fixed processing, i.e. instantaneous ambiguity resolution based on LAMBDA and FFRatio test using a fixed failure rate of 0.001 . Figure 93.4 (top)depicts the outcomes of the FFRatio test for the ionosphere-fixed epoch-by-epoch processing. For $4.5 \%$ of the 2,880
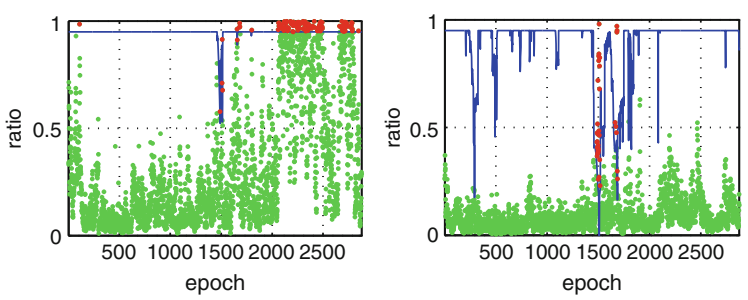

Fig. 93.4 Ratio vs. critical value for the ionosphere-fixed (left) and ionosphere-weighted (right) epoch-by-epoch processing of the $11-\mathrm{km}$ baseline data. If the ratio exceeds the critical value (blue), it is marked red, otherwise green

epochs the FFRatio tests exceeds its critical value, and these rejections are mainly occurring from epoch 2,000 towards the end of the day. For these epochs in Fig. 93.3 (top left) it can be seen that there are large DD ionospheric delays, exceeding $10 \mathrm{~cm}$ in absolute value. Further inspection of the integer solutions revealed that for $0.8 \%$ of the epochs the FFRatio tests is rejected unnecessarily; the integer solutions for these epochs actually correspond to the correct ones. Even more severe, for $7.0 \%$ of the epochs the FFRatio test turned out to accept the wrong integer solution! And this is in complete disagreement with the fixed failure rate set to execute the FFRatio tests of $0.1 \%$. In this context it is emphasized that the performance of FFRatio test depends on the correctness of the underlying models. Hence, these results demonstrate that the traditional ionosphere-fixed model is not suitable for the current baseline as due to the 
significant differential ionospheric delays for a considerable part of the day.

In a next step, the $11-\mathrm{km}$ baseline data have been reprocessed but including the ionospheric corrections using the ionosphere-weighted model and an undifferenced ionospheric standard deviation of $5 \mathrm{~mm}$. Figure 93.4 (right) shows the results of the FFRatio tests for this processing. Comparison with Fig. 93.4 (left) not only demonstrates that the ratios are generally lower, but also the critical values differ between both processing strategies, as they are depending on the choice of model. Now the FFRatio test is rejected for $1.3 \%$ of the epochs. Like in the ionosphere-fixed processing, in $0.8 \%$ of the cases the FFRatio test is rejected unnecessarily. In contrast to the ionosphere-fixed processing, for this ionosphereweighted processing there are no longer epochs with wrongly accepted integer solutions, and this is in agreement with the fixed failure rate, which accepts at most $0.1 \%$ wrong solutions. This result demonstrates that the FFRatio test performs adequately when the processing model underlying is correct. With respect to the success rate (epochs with correctly accepted integer solutions), this is $88.5 \%$ for the ionosphere-fixed processing, but increases to $98.7 \%$ in case of an ionosphere-weighted processing.

\subsubsection{Medium-Baseline Performance}

For the processing of the $38 \mathrm{-km}$ baseline the ionosphere-weighted model is applied using the ionospheric corrections from the network assuming an undifferenced standard deviation of $1 \mathrm{~cm}$. Figure 93.5 shows for the 2,880 epochs the ratios vs. their critical values applying the FFRatio test. The FFRatio tests fails for $3.8 \%$ of the epochs, which are almost all false alarms, since for $3.5 \%$ of the day the FFRatio test is rejected unnecessarily. For 4 epochs during the day the wrong integer ambiguities are estimated, which corresponds to a failure rate of $0.1 \%$. This percentage is in good agreement with the a priori set fixed failure rate in order to execute the FFRatio tests, as it should be when the underlying model is appropriate. For this processing the correct integer solutions were accepted for $96.1 \%$ of the epochs.

Table 93.1 summarizes the empirical failure rates (wrongly accepted epochs), success rates (correctly

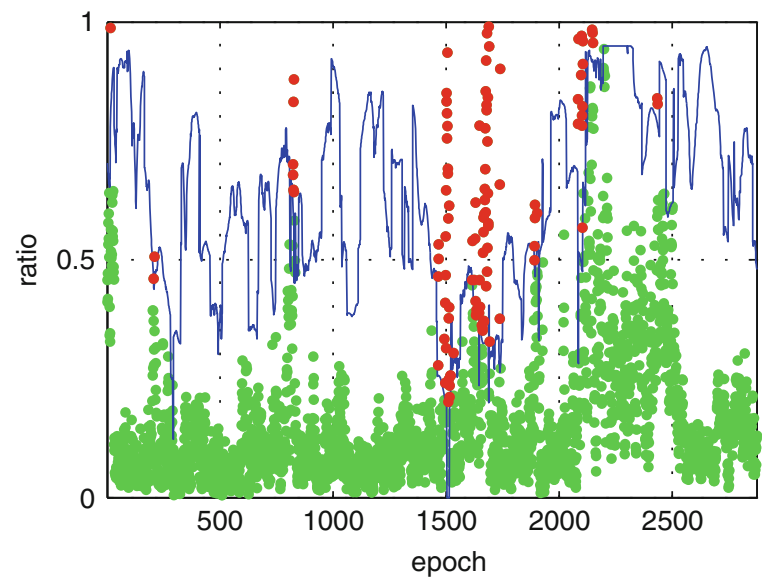

Fig. 93.5 Ratio vs. critical value for the ionosphere-weighted epoch-by-epoch processing of the $38-\mathrm{km}$ baseline data. If the ratio exceeds the critical value (blue), it is marked red, otherwise green

Table 93.1 Empirical instantaneous ambiguity resolution probabilities (using a fixed failure rate of $0.1 \%$ )

\begin{tabular}{lccc}
\hline & $\begin{array}{l}\text { 11-km } \\
\text { ionosphere- } \\
\text { fixed }(\%)\end{array}$ & $\begin{array}{l}11-\mathrm{km} \\
\text { ionosphere- } \\
\text { weighted }(\%)\end{array}$ & $\begin{array}{l}38-\mathrm{km} \\
\text { ionosphere- } \\
\text { weighted }(\%)\end{array}$ \\
\hline Failure & 7.0 & 0 & 0.1 \\
\hline Success & 88.5 & 98.7 & 96.1 \\
\hline False alarm & 0.8 & 0.8 & 3.5 \\
\hline
\end{tabular}

Table 93.2 Empirical instantaneous 95\% horizontal and vertical position errors

\begin{tabular}{llll}
\hline & $\begin{array}{l}11-\mathrm{km} \\
\text { ionosphere- } \\
\text { fixed }\end{array}$ & $\begin{array}{l}11-\mathrm{km} \\
\text { ionosphere- } \\
\text { weighted }\end{array}$ & $\begin{array}{l}38-\mathrm{km} \\
\text { ionosphere- } \\
\text { weighted }\end{array}$ \\
\hline $95 \%$ HPE & $60.6 \mathrm{~cm}$ & $1.7 \mathrm{~cm}$ & $2.7 \mathrm{~cm}$ \\
\hline $95 \%$ VPE & $74.8 \mathrm{~cm}$ & $3.9 \mathrm{~cm}$ & $6.2 \mathrm{~cm}$ \\
\hline
\end{tabular}

accepted epochs) and false alarm rates (wrongly rejected epochs) of the processing strategies applied to the $11-\mathrm{km}$ and $38-\mathrm{km}$ baselines. It is noted that the 'correct rejection rate' then follows from subtracting the first three rates from $100 \%$.

Table 93.2 summarizes the empirical 95\% Horizontal and Vertical Position Errors (HPE and VPE) corresponding to the processing strategies applied to the $11-\mathrm{km}$ and $38-\mathrm{km}$ baselines. These position errors are computed using the precisely known coordinates of stations FXHS and WLSN. The large position errors for the ionosphere-fixed processing are due to the 
many wrong ambiguity fixes. The position errors for both ionosphere-weighted strategies are at sub-dm level.

\section{Conclusions}

Multi-Carrier Ambiguity Resolution (MCAR) for GNSS-RTK applications should be a combination of LAMBDA and the Fixed Failure-rate (FF) Ratio test. While the LAMBDA method optimizes the success of correct integer estimation, the FFRatio test enables the user to have control of the rate of wrong fixes, this in contrast to the traditional ratio tests with fixed threshold values. However, it was shown that successful performance of this FFRatio Test for short to medium distance GPS applications depends on correctness of underlying model. In case the ionospheric delays cannot be neglected, even for short $(\sim 10 \mathrm{~km})$ baselines, accurate network ionospheric corrections should be applied, while their uncertainty should be modeled appropriately through the ionosphere-weighted model.

Acknowledgements Professor Peter J.G. Teunissen is the recipient of an Australian Research Council Federation Fellowship (project number FF0883188). This support is greatly acknowledged. The research of Sandra Verhagen is supported by the Dutch Technology Foundation STW, applied science division of NWO and the Technology Program of the Dutch Ministry of Economic Affairs.

\section{References}

Hernández-Pajares M, Juan JM, Sanz J, Orús R, GarcíaRodríguez A and Colombo O (2004) Wide Area Real-time Kinematics with Galileo and GPS signals, Proc. ION-GNSS 2004, Long Beach, CA, 21-24 Sep. 2004, 2541-2554

Leick A (2003) GPS satellite surveying, 3rd edn. Wiley, New York

Odijk D (1999) Stochastic modelling of the ionosphere for fast GPS ambiguity resolution. Geodesy beyond 2000. The challenges of the first decade, IAG General Assembly, Vol. 121, Birmingham, July 19-30 (1999), pp. 387-392

SCIGN (2008): http://www.scign.org

Teunissen PJG (1995) The least-squares ambiguity decorrelation adjustment: a method for fast GPS integer ambiguity estimation. J Geod 70:65-82

Teunissen PJG (2005) Integer aperture bootstrapping: a new GNSS ambiguity estimator with controllable fail-rate. J Geod 79:389-397

Teunissen PJG, Verhagen S (2009) The GNSS ambiguity ratiotest revisited: a better way of using it. Surv Rev 41 (312):138-151

Vollath U, Deking A, Landau H, Pagels C, and Wagner B (2000) Multi-base RTK positioning using Virtual Reference Stations. Proc. ION GPS-2000, Salt Lake City, UT, 19-22 September 2000 